\title{
The Role of the Neighborhood Social Environment in Physical Activity among Hispanic Children: Moderation by Cultural Factors and Mediation by Neighborhood Norms
}

\author{
Yeonwoo Kim ${ }^{1}$, Lorrene Ritchie ${ }^{2}\left(\mathbb{D}\right.$, Andrew Landgraf ${ }^{3}$, Rebecca E. Hasson ${ }^{4}(\mathbb{C}$ \\ and Natalie Colabianchi ${ }^{4,5, *}$ \\ 1 Department of Kinesiology, The University of Texas at Arlington, Arlington, TX 76010, USA; \\ yeonwoo.kim@uta.edu \\ 2 Nutrition Policy Institute, Division of Agriculture and Natural Resources, University of California, \\ Oakland, CA 94607, USA; lritchie@ucanr.edu \\ 3 Battelle Memorial Institute, Columbus, OH 43201, USA; andland@gmail.com \\ 4 School of Kinesiology, University of Michigan, Ann Arbor, MI 48109, USA; hassonr@umich.edu \\ 5 Institute for Social Research, University of Michigan, Ann Arbor, MI 48104, USA \\ * Correspondence: colabian@umich.edu; Tel.: +1-(734)-764-4765
}

Received: 29 October 2020; Accepted: 16 December 2020; Published: 19 December 2020

\begin{abstract}
Little is known about how the neighborhood social environment (e.g., safety, crime, traffic) impacts child physical activity. We examine the mechanism by which the neighborhood social environment is associated with child physical activity, moderated by individual-level cultural factors (e.g., language at home, immigrant generation) and mediated by neighborhood physical activity-related social norms (e.g., seeing walkers in the neighborhood). Data included 2749 nonHispanic White and Hispanic children from the Healthy Communities Study. Multilevel regression was performed. The neighborhood social environment was not associated with physical activity in the full sample. However, Hispanic children speaking both English and Spanish and first- or second-generation Hispanic children engaged in more physical activity when the quality of the neighborhood social environment was higher $(b=1.60, p<0.001$ for Hispanic children speaking English and Spanish; $b=2.03, p<0.01$ for first-generation Hispanic children; $b=1.29, p<0.01$ for second-generation Hispanic children). Neighborhood physical activity-related social norms mediated the association between the neighborhood social environment and physical activity among Hispanic children speaking English and Spanish $(b=0.33, p<0.001)$ and second-generation Hispanic children $(b=0.40, p<0.001)$. Findings suggest heterogeneity in how neighborhood social environments impact physical activity by cultural factors. Health promotion programs may need to enhance neighborhood social environments to increase Hispanic children's physical activity.
\end{abstract}

Keywords: social environment; cultural factors; physical activity; neighborhood social norms

\section{Introduction}

Lack of physical activity among children is a serious public health issue in the United States (US). Only four in ten US children engage in the recommended amount of physical activity [1], and $11 \%$ of children do not participate in any vigorous physical activity in a given week [2]. When examining physical inactivity by ethnicity, Hispanic children are the most inactive; $15 \%$ of Hispanic children with US-born parents, and 17-23\% of Hispanic children with immigrant parents do not engage in any vigorous physical activity (vs. 9\% of non-Hispanic White children) [2]. A low level of physical activity 
among Hispanic children contributes to their high prevalence of overweight and obesity (over 23\%) [3], a prevalence that has continued to increase despite recent declines among non-Hispanic White children [4]. As Hispanic populations are the largest ethnic minority group in the US, representing $29 \%$ of children [5], and will account for $32 \%$ of the total child population in 2060 [6], attention has recently increased to identify factors related to physical inactivity in Hispanic children.

\subsection{Neighborhood Impacts on Child Physical Activity}

The neighborhood environment can affect child physical activity levels, for example, by influencing whether they can safely walk to a park or playground [7-10]. In addition, children may observe more residents engaging in physical activity when living in safe and secure neighborhoods. The active lifestyle of residents attributes to social norms, which may encourage children to engage in physical activity [11]. In other words, living in safe and secure neighborhoods may positively affect child physical activity, mediated by social norms around engaging in activity. Empirical studies that have investigated the association between the neighborhood social environment (e.g., safety, crime, traffic) and child physical activity have reported mixed results $[8,9,12-17]$. The mixed findings may partly be explained by limited consideration of moderating factors such as individual-level ethnicity and cultural factors (e.g., immigrant generation, language spoken at home), in addition to variation in survey designs, sample characteristics, and measurements. Thus, the present study examines the moderating effect of individual-level cultural factors in the association between the neighborhood social environment and physical activity. In addition, given that no prior studies have investigated potential mediating mechanisms underlying an association, this study investigates the mediating role of neighborhood physical activity-related social norms in the association between the social environment and child physical activity.

\subsection{Considering Cultural Factors to Understand How Neighborhood Impacts Physical Activity}

Several studies have examined individual-level cultural factors, such as language spoken at home and immigrant generation, as a correlate of physical activity in Hispanic children [2,17-23]. Theoretically, two contrasting mechanisms have been proposed to examine the association between cultural factors and physical activity in Hispanic children. On the one hand, as physical activity is often a social behavior for children mostly involving groups of peers [24,25], fewer language barriers and greater exposure to US culture are likely to provide more opportunities to be physically active with peers from diverse cultural backgrounds. On the other hand, greater exposure to US culture may increase the risk of inactivity for Hispanic children given acculturation-related stress (leading to unhealthy lifestyle) and the high levels of physical inactivity in the US [26-28]. A few empirical studies reported that being first-generation or Spanish speaking is directly associated with less physical activity among Hispanic children after adjusting for parental education and economic level [2,20,21], which supports the first conceptual mechanism (see an exception in [18]).

Cultural factors may interact with social environments to affect child physical activity although to our knowledge, no empirical research has investigated its interaction effect. Past literature showed variation across Hispanic immigrant generations in types of resources that parents use to support their children. For example, parents of first-generation Hispanic children used informal support for their children, while parents of third-generation Hispanic children used formal and structural support for their children [29]. Living in a high-quality social environment may be an important informal support for Spanish-speaking Hispanic children and first- or second-generation Hispanic children. Safe and supportive environments may lead to more physical activity within the neighborhood thereby exposing them to visual cues of neighbors and peers being physically active in their neighborhood. Also, Spanish-speaking or first- or second-generation Hispanic families can face practical and cultural challenges to navigating and utilizing physical activity resources outside their neighborhood [21,30-32]. Thus, living in a health-promoting neighborhood may provide more beneficial informal support for 
them in comparison to non-Hispanic White children and Hispanic children from English-speaking or third-generation families who may be more likely to use formal and structural resources.

\subsection{The Present Study}

The present study extends past research by examining moderating and mediating mechanisms underlying the association between the social environment and physical activity in non-Hispanic White and Hispanic children. We hypothesized that (1) the protective role of the social environment in physical activity would be stronger for Spanish-speaking or first- or second-generation Hispanic children compared to non-Hispanic White children and English-speaking or third-generation Hispanic children; and (2) active and healthy social norms in the neighborhood would mediate the association between the social environment and physical activity.

\section{Methods}

\subsection{Participants}

We used data from the Healthy Communities Study (HCS; 2013-2015). The Battelle Memorial Institute and its academic partners (University of California at Berkeley, University of South Carolina, and University of Kansas) designed and conducted the HCS, an observational study designed to examine community impacts on child obesity in the US. The HCS first recruited a probability-based sample of 102 communities (defined by high school catchment areas) by stratifying by race, ethnicity, income, region, and a pre-selection score of program and policy intensity [33]. The HCS added 28 communities that were purposefully selected for their childhood obesity prevention efforts [33]. The HCS recruited child-parent/guardian dyads $(n=5138)$ from elementary and middle schools located in 130 communities. Trained personnel collected the data from participants at home visits including socio-demographic information, physical activity behavior, and child perception of their social environment. The respondent was determined by child age. Specifically, child age was recorded, and the Computer-Assisted Interview system guided who was to answer each question. Additional details about the HCS have been reported elsewhere [33].

Our research team obtained access to the HCS data under contract and extended the HCS data by linking the American Community Survey 2009-2013 data to the HCS participant data. Our sample included non-Hispanic White children (US-born and English-speaking at home) and Hispanic children who lived at their current address for 1 year or more and had a geocoded address $(n=3472)$. We excluded Hispanic children who used language other than English and/or Spanish due to the small sample size $(n=9)$, resulting in 3463 children. Participants were excluded if they had missing data on: physical activity $(n=193)$, child's or parents' country of birth $(n=269)$, two or more items for child perception of the social environment $(n=39)$, neighborhood physical activity-related social norms ( $n=47)$, maximum parent education $(n=14)$, and household income $(n=142)$. The resulting final sample included 2749 child-adult respondent dyads. Compared with respondents remaining in the study, those excluded were more likely to be older, Hispanic ethnicity, in families with lower socioeconomic status, and have lower levels of child-perceived social environment. There were no significant differences $(p>0.05)$ between included and excluded samples in physical activity level and neighborhood physical activity-related norms. This study was reviewed by the University of Michigan Institutional Review Board and the Battelle Memorial Institute Institutional Review Board (The University of Michigan's IRB HUM number is HUM00138571. The Battelle Memorial Institute's IRB number is IRB 0677-100112203 Rev 0.0). 


\subsection{Measures}

\subsubsection{Outcome Measure}

Physical activity was measured using the 7-day Physical Activity Behavior Recall (PABR-7) instrument designed to elicit participation in 14 types of activities in the prior week. We used six of the 14 activity types including pick-up sports (e.g., basketball, football), non-school sports, physically active games, swimming, outdoor/adventure activities (e.g., rock climbing), and walk/bike for fun/exercise. We excluded the other eight activity types that were performed at school because we focused on associations with residential neighborhood-based physical activity. Using a computer-assisted interview, respondents indicated the days on which the child did each activity during the past week. These questions were answered by an adult respondent (mostly parent) for children aged 4-8; otherwise by the child participant for children aged 9-15. Physical activity was examined as a continuous variable (sum of number of times in the past week for all 6 physical activities). Additional details on physical activity measures are available elsewhere [34].

\subsubsection{Independent Variable}

Child perception of the social environment was included as an independent variable. Children responded to a four-item scale each with four response options (disagree a lot to agree a lot): "It is safe to walk or jog in the neighborhood during the day," "There is so much traffic that it makes it hard to walk in the neighborhood (reverse coded)," "There is a lot of crime in the neighborhood (reverse coded)," and "There are lots of loose or scary dogs in the neighborhood (reverse coded)." We calculated the average of four items (range 1-4 points) allowing for one item to be missing. For children aged $4-11$, an adult respondent (mostly parent) assisted the child to answer the questions. Higher scores represent a better social environment (Cronbach's alpha $=0.60$ ).

\subsubsection{Moderators}

Moderators included individual-level cultural factors, which were measured using ethnicity, language at home and immigrant generation. First, language at home was measured based on Hispanic identification and language spoken at home. Adult respondents were asked the focal child's Hispanic identification (Hispanic or not) and language spoken at home. English-speaking non-Hispanic White children were coded as "English-speaking non-Hispanic White children" $(=0)$. If the Hispanic child spoke only English at home, the child was coded as "Hispanic children speaking English" (=1). If the Hispanic child used English and Spanish equally or more English than Spanish at home, the child was coded as "Hispanic children speaking English and Spanish" (=2). If the Hispanic child spoke Spanish only or more than English, the child was coded as "Hispanic children speaking Spanish" (=3). Second, immigrant generation was measured based on the country of birth for the children and their biological parents. The variable was categorized as "US-born non-Hispanic White children" $(=0)$, "first-generation Hispanic children" (=1), "second-generation Hispanic children" (=2), and "third- or higher-generation Hispanic children" (=3).

\subsubsection{Mediator}

Neighborhood physical activity-related social norms were included as a mediator between the social environment and physical activity. Neighborhood physical activity-related social norms were measured as a continuous variable based on two questions asked of children with four response options (disagree a lot to agree a lot): (1) people in my neighborhood can easily see walkers and bikers on the streets from their homes; and (2) I often see other girls or boys playing outdoors in my neighborhood. 


\subsubsection{Covariates}

Covariates included child age, child sex, biological parents' highest education, annual household income, and neighborhood socioeconomic status. We used the American Community Survey 2009-2013 to obtain the measure of neighborhood socioeconomic status. Neighborhood socioeconomic status was measured by summing the $\mathrm{z}$ scores of variables representing income, housing values, education, and occupation at the block group level and then calculating the weighted average of the summated $\mathrm{z}$ scores within $1 \mathrm{~km}$ of the participant's residence [35,36]. We categorized neighborhoods into three groups using tertiles of weighted neighborhood socioeconomic status score.

\subsection{Analysis}

Descriptive statistics and analysis of variance (ANOVA) were used to explore the sample characteristics and the bivariate relationship between sociodemographic characteristics and child physical activity level. In subsequent regression analysis, multilevel modeling was used given that the data from the HCS were clustered at the community level (intraclass correlation for physical activity = $6.46 \%$ ). We first conducted a multilevel linear regression model to assess associations between the social environment and physical activity in non-Hispanic White and Hispanic children. Second, we tested two interaction terms, one at a time, on the association between the social environment and physical activity: (1) interaction term of social environment and language at home and (2) interaction term of the social environment and immigrant generation. Due to the significant differences of some variables between the excluded sample and the included sample, we conducted multiple imputation for missing data among 3463 children and assessed multilevel linear regression models as a sensitivity analysis. Lastly, we conducted a structural equation model using bootstrap analyses (500 bootstrap samples) to test the mediation effect of neighborhood physical activity-related social norms in the association between the social environment and physical activity [37]. We decomposed the total, direct, and indirect effects to further understand the direction and extent of the mediation effect. When a cultural factor was a significant moderator in the association between the social environment and physical activity, the mediation analysis was conducted in the groups where the social environment was significantly associated with physical activity. Analyses were performed using Stata Version 15 (StataCorp LLC, College Station, TX, USA).

\section{Results}

Sample characteristics are shown in Table 1 . In the analytical sample, $44 \%$ of children identified as English-speaking non-Hispanic White children, 36\% identified as Hispanic children speaking English and Spanish, 14\% identified as Hispanic children speaking Spanish, and 6\% identified as Hispanic children speaking English at home. Regarding immigrant generation, $44 \%$ of children were second-generation Hispanic children, 7\% identified as first-generation Hispanic children, and 6\% identified third- or higher-generation Hispanic children. Children reported participating in any of six physical activity types 7.8 times during the past week on average.

Table 2 present the results of bivariate analyses. Bivariate statistics showed a significant difference in physical activity level between the following groups: (1) children aged 10-12 years $(M=8.2)$ and children aged 13-15 years (Mean =7.1), (2) boys (Mean = 8.1) and girls (Mean =7.4), (3) English-speaking non-Hispanic White children (Mean $=8.4$ ) and Hispanic children speaking English and Spanish or speaking Spanish (Mean = 7.2 and 7.1, respectively), (4) US-born non-Hispanic White children $($ Mean $=8.4)$ and second generation Hispanic children (Mean = 7.2), $(5)$ children of parents with some college (Mean $=8.0$ ) and children of parents with less than high school (Mean = 7.0), (6) children of parents with college graduate or above (Mean $=8.3$ ) and children of parents with less than high school (Mean $=7.0$ ), (7) children of families with annual income $\$ 75,001$ or above (Mean $=8.4$ ) and children of families with annual income less than $\$ 20,000$ (Mean $=7.3$ ), $\$ 20,000-35,000$ (Mean = 7.3), 
and $\$ 35,001-50,000$ (Mean = 7.3), and (8) children living in high SES neighborhoods (Mean = 8.3) and children living in low or moderate SES neighborhoods (Mean $=7.6$ and 7.3, respectively).

Table 1. Sample characteristics, $\mathrm{n}=2749$.

\begin{tabular}{|c|c|c|}
\hline Characteristic & $\mathbf{n}$ & $\%$ \\
\hline Frequency of physical activity (Mean times per week \pm SD) & \multicolumn{2}{|c|}{$7.8 \pm 6.1$} \\
\hline \multicolumn{3}{|l|}{ Child age } \\
\hline $4-6$ years & 528 & 19.2 \\
\hline $7-9$ years & 978 & 35.6 \\
\hline 10-12 years & 863 & 31.4 \\
\hline $13-15$ years & 380 & 13.8 \\
\hline \multicolumn{3}{|l|}{ Child gender } \\
\hline Boy & 1400 & 50.9 \\
\hline Girl & 1349 & 49.1 \\
\hline Frequency of physical activity (Mean times per week \pm SD) & \multicolumn{2}{|c|}{$7.8 \pm 6.1$} \\
\hline \multicolumn{3}{|l|}{ Child language at home } \\
\hline Non-Hispanic White ${ }^{\text {a }}$ & 1211 & 44.1 \\
\hline Hispanics speaking English & 160 & 5.8 \\
\hline Hispanics speaking English and Spanish & 987 & 35.9 \\
\hline Hispanics speaking Spanish & 391 & 14.2 \\
\hline \multicolumn{3}{|l|}{ Child immigrant generation } \\
\hline Non-Hispanic White ${ }^{a}$ & 1211 & 44.1 \\
\hline First generation Hispanics & 180 & 6.6 \\
\hline Second generation Hispanics & 1200 & 43.7 \\
\hline Third or higher generation Hispanics & 158 & 5.8 \\
\hline \multicolumn{3}{|l|}{ Maximum parent education } \\
\hline Less than high school & 742 & 27.0 \\
\hline High-school graduate & 455 & 16.6 \\
\hline Some college & 572 & 20.8 \\
\hline College graduate or above & 980 & 35.7 \\
\hline \multicolumn{3}{|l|}{ Annual household income } \\
\hline Less than $\$ 20,000$ & 634 & 23.1 \\
\hline$\$ 20,000-35,000$ & 633 & 23.0 \\
\hline$\$ 35,001-50,000$ & 346 & 12.6 \\
\hline$\$ 50,001-75,000$ & 303 & 11.0 \\
\hline$\$ 75,001$ or above & 833 & 30.3 \\
\hline Child-perceived social environment $($ Mean \pm SD) $b$ & \multicolumn{2}{|c|}{$2.5 \pm 0.7$} \\
\hline Neighborhood physical activity social norms (Mean \pm SD) $b$ & \multicolumn{2}{|c|}{$3.1 \pm 0.8$} \\
\hline \multicolumn{3}{|l|}{ Neighborhood socioeconomic status ${ }^{\mathrm{c}}$} \\
\hline Low SES $(<-0.40)$ & 902 & 32.8 \\
\hline Moderate SES ( -0.40 to -0.02$)$ & 841 & 30.6 \\
\hline High SES $(>-0.02)$ & 1006 & 36.6 \\
\hline \multicolumn{3}{|c|}{$\begin{array}{l}\text { a English-speaking, US-born non-Hispanic White children. }{ }^{\mathrm{b}} \text { Social environment was measured based on a four-item } \\
\text { scale on neighborhood safety, traffic, and crime. Neighborhood physical activity-related social norms were measured } \\
\text { based on two indicators of seeing others being active in the neighborhood. Higher scores indicate higher quality } \\
\text { social environment or more physical activity-promoting norms. }{ }^{c} \text { Neighborhood socioeconomic status was based on } \\
\text { American Community Survey } 5 \text { year estimates of } 2009-2013 \text {. We categorized this variable into three groups by } \\
\text { tertiles. Higher tertiles represent higher socioeconomic status. }\end{array}$} \\
\hline
\end{tabular}

Table 3 presents associations of the social environment and language at home with physical activity. The social environment was not significantly associated with physical activity in the overall sample. Hispanic children speaking English and Spanish or speaking Spanish had less physical activity than non-Hispanic White children $(b=-1.38$ and -1.66 , respectively, $p<0.001)$ after controlling for child age and gender (Model 1). With the inclusion of parental education, household income, social environment, and neighborhood socioeconomic status in the model (Model 2), the associations remained significant 
$(b=-0.94, p<0.05$ and $b=-1.24, p<0.01$ respectively). There was no statistical difference in physical activity between Hispanic children speaking English and non-Hispanic White children $(b=-0.62$, $p>0.05)$. In Model 3, there was a significant interaction effect of speaking English and Spanish at home (vs. non-Hispanic White children) in the association between the social environment and physical activity $(b=1.60, p<0.001)$. As shown in Figure 1 , the association between the social environment and physical activity was positive for Hispanic children speaking English and Spanish whereas the association was negative and flatter for non-Hispanic White children. Although it was not significant at alpha $=0.05$ for Hispanic children speaking Spanish, the association between the social environment and physical activity was marginally significant and positive for Hispanic children speaking Spanish in comparison to non-Hispanic White children $(b=0.92, p<0.10)$.

Table 2. Bivariate statistics, $\mathrm{n}=2749$.

\begin{tabular}{|c|c|c|}
\hline \multirow{2}{*}{ Characteristics } & \multicolumn{2}{|c|}{ Average Frequency of Physical Activity } \\
\hline & Mean $\pm \mathrm{SD}^{\mathrm{c}}$ & F (t-Value) \\
\hline Child age & & $3.64 *$ \\
\hline 4-6 years & $7.5 \pm 5.4$ & \\
\hline 7-9 years & $7.8 \pm 5.9$ & \\
\hline 10-12 years & $8.2 \pm 6.5$ & \\
\hline $13-15$ years & $7.1 \pm 6.0$ & \\
\hline Child gender & & $\left(\mathrm{t}=2.86^{* *}\right)$ \\
\hline Boy & $8.1 \pm 0.2$ & \\
\hline Girl & $7.4 \pm 0.2$ & \\
\hline Child language at home & & $8.71^{* * *}$ \\
\hline Non-Hispanic White ${ }^{a}$ & $8.4 \pm 6.0$ & \\
\hline Hispanics speaking English & $7.8 \pm 5.8$ & \\
\hline Hispanics speaking English and Spanish & $7.2 \pm 6.1$ & \\
\hline Hispanics speaking Spanish & $7.1 \pm 6.1$ & \\
\hline Child immigrant generation & & $8.96^{* * *}$ \\
\hline Non-Hispanic White ${ }^{a}$ & $8.4 \pm 6.0$ & \\
\hline First generation Hispanics & $7.2 \pm 6.4$ & \\
\hline Second generation Hispanics & $7.2 \pm 6.1$ & \\
\hline Third or higher generation Hispanics & $8.0 \pm 5.7$ & \\
\hline Maximum parent education & & $6.85^{* * *}$ \\
\hline Less than high school & $7.0 \pm 6.1$ & \\
\hline High-school graduate & $7.7 \pm 6.4$ & \\
\hline Some college & $8.0 \pm 6.2$ & \\
\hline College graduate or above & $8.3 \pm 5.7$ & \\
\hline Annual household income & & $5.57^{* * *}$ \\
\hline Less than $\$ 20,000$ & $7.3 \pm 6.4$ & \\
\hline$\$ 20,000-35,000$ & $7.3 \pm 6.0$ & \\
\hline$\$ 35,001-50,000$ & $7.3 \pm 5.9$ & \\
\hline$\$ 50,001-75,000$ & $8.3 \pm 6.1$ & \\
\hline$\$ 75,001$ or above & $8.4 \pm 5.7$ & \\
\hline Neighborhood socioeconomic status ${ }^{b}$ & & $5.97^{* *}$ \\
\hline Low SES $(<-0.40)$ & $7.6 \pm 6.3$ & \\
\hline Moderate SES ( -0.40 to -0.02$)$ & $7.3 \pm 6.0$ & \\
\hline High SES (>-0.02) & $8.3 \pm 5.8$ & \\
\hline
\end{tabular}

\footnotetext{
a English-speaking, US-born non-Hispanic White children. ${ }^{\mathrm{b}}$ Neighborhood socioeconomic status was based on American Community Survey 5 year estimates of 2009-2013. We categorized this variable into three groups by tertiles. Higher tertiles represent higher socioeconomic status. ${ }^{\mathrm{c}} \mathrm{SD}$ indicates standard deviation. ${ }^{*} p<0.05$; ** $p<0.01 ; * * * 0<0.001$.
} 


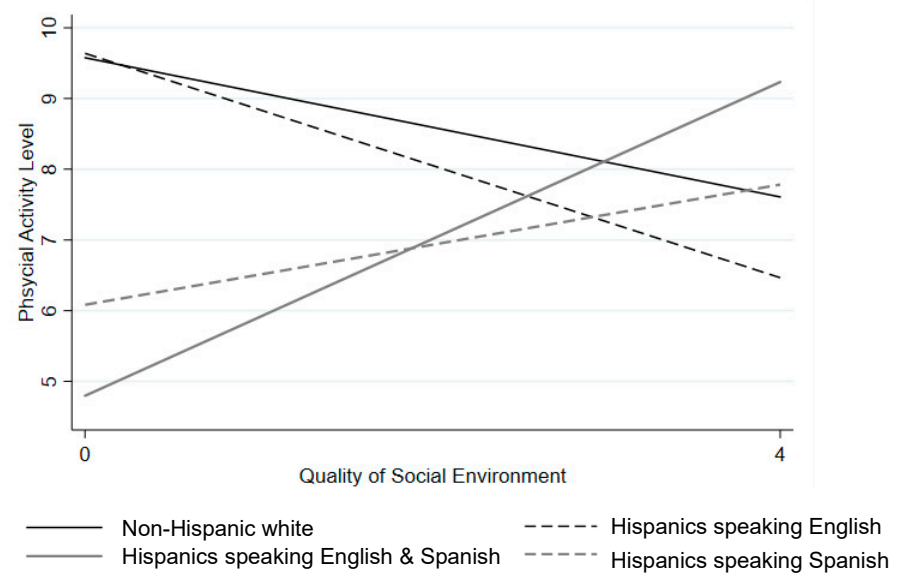

Figure 1. Predicted physical activity associated with the social environment by child language at home, as shown in Table 3, Model 3, setting all other variables within the model at their average value. Note. The social environment was measured based on a four-item scale on neighborhood safety, traffic, and crime. Higher scores indicate higher quality social environment.

Table 4 presents associations of the social environment and immigrant generation with physical activity. The social environment was not significantly associated with physical activity in the overall sample. First or second-generation Hispanics had less physical activity than US-born non-Hispanic White children after adjusting for child age and gender $(b=-1.53, p<0.01$ and $b=-1.46, p<0.001$ respectively). The associations remained significant $(b=-1.12, p<0.05$ for first-generation Hispanics and $b=-1.03, p<0.01$ for second-generation Hispanics) when parental education, household income, social environment, and neighborhood socioeconomic status were added to the model (Model 2). There was no significant difference in physical activity between third- or higher-generation Hispanics and non-Hispanic White children. In Model 3, significant interactions were found between the social environment and child immigrant generation in the association with physical activity. As shown in Figure 2, the association between the social environment and physical activity was positive for first- and second-generation Hispanic children $(b=2.03, p<0.01$ and $b=1.29, p<0.01$, respectively), whereas the association was negative and flatter for non-Hispanic White children.

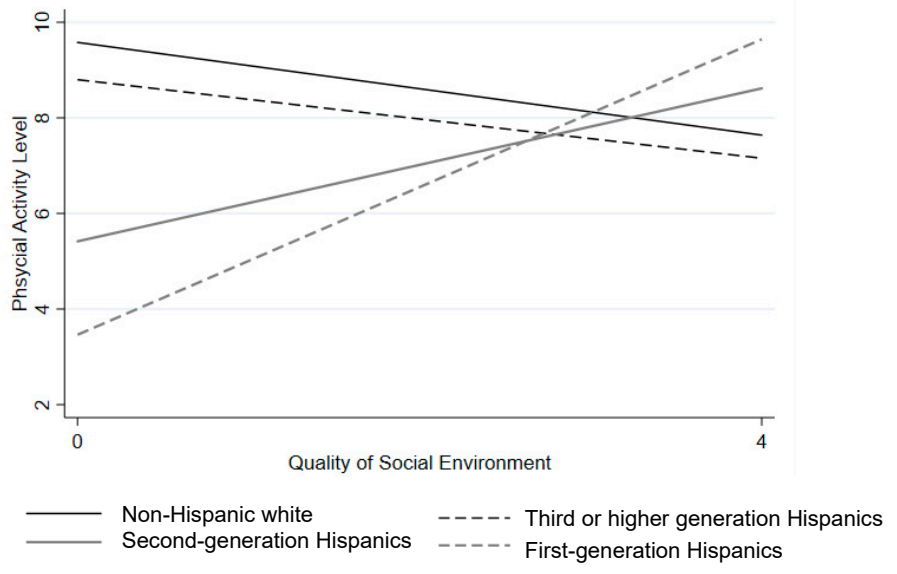

Figure 2. Predicted physical activity associated with the social environment by child immigrant generation, as shown in Table 4, Model 3, setting all other variables within the model at their average value. Note. The social environment was measured based on a four-item scale on neighborhood safety, traffic, and crime. Higher scores indicate higher quality social environment. 
Table 3. Results of multilevel modeling assessing associations between child language at home and physical activity (times per week), $\mathrm{n}=2749$.

\begin{tabular}{|c|c|c|c|c|c|c|c|c|c|}
\hline \multirow{2}{*}{ Predictors } & \multicolumn{3}{|c|}{ Model 1} & \multicolumn{3}{|c|}{ Model 2} & \multicolumn{3}{|c|}{ Model 3} \\
\hline & B & SE & $95 \% \mathrm{CI}$ & B & SE & $95 \% \mathrm{CI}$ & B & SE & $95 \% \mathrm{CI}$ \\
\hline \multicolumn{10}{|l|}{ Child age } \\
\hline 7-9 years & 0.36 & 0.32 & $-0.26,0.99$ & 0.37 & 0.32 & $-0.25,1.00$ & 0.41 & 0.32 & $-0.22,1.03$ \\
\hline $10-12$ years & $0.78 *$ & 0.33 & $0.13,1.42$ & $0.74 *$ & 0.33 & $0.09,1.39$ & $0.78 *$ & 0.33 & $0.13,1.43$ \\
\hline $13-15$ years & -0.45 & 0.41 & $-1.25,0.34$ & -0.51 & 0.41 & $-1.31,0.29$ & -0.51 & 0.41 & $-1.30,0.29$ \\
\hline $\begin{array}{l}\text { Boy (reference) } \\
\text { Girl }\end{array}$ & $-0.65 * *$ & 0.22 & $-1.09,-0.21$ & -0.65 ** & \multicolumn{4}{|c|}{ Boy (reference) } & \\
\hline \multicolumn{10}{|l|}{ Child language at home } \\
\hline \multicolumn{10}{|l|}{ Non-Hispanic White ${ }^{a}$ (reference) } \\
\hline Hispanics speaking English & -0.74 & 0.52 & $-1.76,0.27$ & -0.62 & 0.53 & $-1.65,0.41$ & 0.62 & 1.89 & $-3.64,3.76$ \\
\hline Hispanics speaking English and Spanish & $-1.38^{* * *}$ & 0.31 & $-1.98,-0.77$ & $-0.94 *$ & 0.37 & $-1.68,-0.21$ & $-4.78^{* * *}$ & 1.05 & $-6.84,-2.72$ \\
\hline Hispanics speaking Spanish & $-1.66^{* * *}$ & 0.39 & $-2.43,-0.89$ & $-1.24^{* *}$ & 0.45 & $-2.11,-0.36$ & $-3.49 *$ & 1.39 & $-6.22,-0.77$ \\
\hline \multicolumn{10}{|l|}{ Maximum parent education } \\
\hline Less than high school & & & & -0.28 & 0.43 & $-1.12,0.57$ & -0.35 & 0.43 & $-1.19,0.49$ \\
\hline High-school graduate & & & & 0.36 & 0.43 & $-0.48,1.20$ & 0.24 & 0.43 & $-0.60,1.08$ \\
\hline Some college & & & & 0.18 & 0.36 & $-0.54,0.89$ & 0.09 & 0.36 & $-0.62,0.80$ \\
\hline \multicolumn{10}{|l|}{ College graduate or above (reference) } \\
\hline \multicolumn{10}{|l|}{ Annual household income } \\
\hline \multicolumn{10}{|l|}{ Less than $\$ 20,000$ (reference) } \\
\hline$\$ 20,000-35,000$ & & & & 0.03 & 0.33 & $-0.63,0.68$ & 0.01 & 0.33 & $-0.64,0.67$ \\
\hline$\$ 35,001-50,000$ & & & & -0.22 & 0.41 & $-1.01,0.58$ & -0.16 & 0.40 & $-0.96,0.63$ \\
\hline$\$ 50,001-75,000$ & & & & 0.43 & 0.45 & $-0.46,1.32$ & 0.44 & 0.45 & $-0.44,1.33$ \\
\hline$\$ 75,001$ or above & & & & 0.36 & 0.44 & $-0.50,1.22$ & 0.49 & 0.44 & $-0.37,1.35$ \\
\hline Child-perceived social environment ${ }^{b}$ & & & & 0.35 & 0.19 & $-0.01,0.72$ & -0.49 & 0.31 & $-1.10,0.12$ \\
\hline \multicolumn{10}{|l|}{ Neighborhood socioeconomic status ${ }^{c}$} \\
\hline \multicolumn{10}{|l|}{ Low SES (reference) } \\
\hline Moderate SES & & & & -0.15 & 0.36 & $-0.85,0.56$ & -0.12 & 0.36 & $-0.82,0.59$ \\
\hline High SES & & & & 0.11 & 0.41 & $-0.68,0.91$ & 0.26 & 0.41 & $-0.54,1.06$ \\
\hline \multicolumn{10}{|l|}{$\begin{array}{l}\text { Child language at home } \times \text { Social environment } \\
\text { Non-Hispanic White }{ }^{\mathrm{a}} \times \text { Social environment (reference) }\end{array}$} \\
\hline \multicolumn{10}{|l|}{ Non-Hispanic White ${ }^{\mathrm{a}} \times$ Social environment (reference) } \\
\hline Hispanics speaking English $\times$ Social environment & & & & & & & -0.30 & 0.72 & $-1.71,1.11$ \\
\hline Hispanics speaking English and Spanish $\times$ Social environment & & & & & & & $1.60^{* * *}$ & 0.40 & $0.81,2.39$ \\
\hline Hispanics speaking Spanish $\times$ Social environment & & & & & & & 0.92 & 0.54 & $-0.15,1.98$ \\
\hline
\end{tabular}


Table 4. Results of multilevel modeling assessing associations between child immigrant generation and physical activity (times per week), $\mathrm{n}=2749$.

\begin{tabular}{|c|c|c|c|c|c|c|c|c|c|}
\hline \multirow{2}{*}{ Predictors } & \multicolumn{3}{|c|}{ Model 1} & \multicolumn{3}{|c|}{ Model 2} & \multicolumn{3}{|c|}{ Model 3} \\
\hline & B & SE & $95 \% \mathrm{CI}$ & B & SE & $95 \% \mathrm{CI}$ & B & SE & $95 \% \mathrm{CI}$ \\
\hline \multicolumn{10}{|l|}{ Child age } \\
\hline \multicolumn{10}{|l|}{$4-6$ years (reference) } \\
\hline 7-9 years & 0.37 & 0.32 & $-0.26,0.99$ & 0.38 & 0.32 & $-0.24,1.01$ & 0.41 & 0.32 & $-0.21,1.03$ \\
\hline $10-12$ years & $0.79 *$ & 0.33 & $0.14,1.43$ & $0.75 *$ & 0.33 & $0.10,1.40$ & $0.79 *$ & 0.33 & $0.15,1.44$ \\
\hline $13-15$ years & -0.42 & 0.41 & $-1.22,0.37$ & -0.47 & 0.41 & $-1.27,0.33$ & -0.46 & 0.41 & $-1.26,0.34$ \\
\hline \multicolumn{10}{|l|}{ Child gender } \\
\hline \multicolumn{10}{|l|}{ Boy (reference) } \\
\hline Girl & $-0.66^{* *}$ & 0.22 & $-1.10,-0.22$ & $-0.65^{* *}$ & 0.22 & $-1.09,-0.21$ & $-0.64 * *$ & 0.22 & $-1.08,-0.20$ \\
\hline \multicolumn{10}{|l|}{ Child immigrant generation } \\
\hline \multicolumn{10}{|l|}{ Non-Hispanic White a (reference) } \\
\hline First generation Hispanics & $-1.53 * *$ & 0.51 & $-2.52,-0.53$ & $-1.12 *$ & 0.54 & $-2.19,-0.05$ & $-6.12 * *$ & 1.86 & $-9.77,-2.46$ \\
\hline Second generation Hispanics & $-1.46^{* * *}$ & 0.30 & $-2.04,-0.87$ & $-1.03 * *$ & 0.36 & $-1.75,-0.32$ & $-4.16^{* * *}$ & 1.02 & $-6.17,-2.16$ \\
\hline Third or higher generation Hispanics & -0.59 & 0.52 & $-1.62,0.43$ & -0.48 & 0.53 & $-1.52,0.56$ & -0.78 & 1.84 & $-4.38,2.82$ \\
\hline \multicolumn{10}{|l|}{ Maximum parent education } \\
\hline Less than high school & & & & -0.27 & 0.43 & $-1.11,0.58$ & -0.36 & 0.43 & $-1.20,0.49$ \\
\hline High-school graduate & & & & 0.35 & 0.43 & $-0.49,1.20$ & 0.23 & 0.43 & $-0.61,1.07$ \\
\hline Some college & & & & 0.16 & 0.36 & $-0.55,0.88$ & 0.07 & 0.36 & $-0.64,0.79$ \\
\hline \multicolumn{10}{|l|}{ College graduate or above (reference) } \\
\hline \multicolumn{10}{|l|}{ Annual household income } \\
\hline \multicolumn{10}{|l|}{ Less than $\$ 20,000$ (reference) } \\
\hline$\$ 20,000-35,000$ & & & & 0.01 & 0.33 & $-0.65,0.66$ & -0.03 & 0.33 & $-0.68,0.63$ \\
\hline$\$ 35,001-50,000$ & & & & -0.21 & 0.41 & $-1.01,0.58$ & -0.20 & 0.41 & $-1.00,0.59$ \\
\hline$\$ 50,001-75,000$ & & & & 0.41 & 0.45 & $-0.48,1.30$ & 0.41 & 0.45 & $-0.48,1.30$ \\
\hline$\$ 75,001$ or above & & & & 0.34 & 0.44 & $-0.52,1.20$ & 0.42 & 0.44 & $-0.44,1.28$ \\
\hline Child-perceived social environment ${ }^{b}$ & & & & 0.35 & 0.19 & $-0.01,0.71$ & -0.48 & 0.31 & $-1.09,0.12$ \\
\hline \multicolumn{10}{|l|}{ Neighborhood socioeconomic status ${ }^{c}$} \\
\hline \multicolumn{10}{|l|}{ Low SES (reference) } \\
\hline Moderate SES & & & & -0.15 & 0.36 & $-0.86,0.55$ & -0.14 & 0.36 & $-0.84,0.57$ \\
\hline High SES & & & & 0.10 & 0.41 & $-0.69,0.90$ & 0.24 & 0.41 & $-0.56,1.04$ \\
\hline \multicolumn{10}{|l|}{ Child immigrant generation $\times$ Social environment } \\
\hline Non-Hispanic White a $\times$ Social environment (reference) & & & & & & & & & \\
\hline First generation Hispanics $\times$ Social environment & & & & & & & $2.03 * *$ & 0.73 & $0.60,3.46$ \\
\hline Second generation Hispanics $\times$ Social environment & & & & & & & $1.29 * *$ & 0.39 & $0.52,2.05$ \\
\hline Third or higher generation Hispanics $\times$ Social environment & & & & & & & 0.07 & 0.71 & $-1.32,1.47$ \\
\hline
\end{tabular}

SES: Socioeconomic status. ${ }^{*} p<0.05,{ }^{* *} p<0.01,{ }^{* * *} p<0.001 .{ }^{\text {a }}$ The reference group is English-speaking, US-born non-Hispanic White children. ${ }^{\mathrm{b}}$ Social environment was measured based on a four-item scale on neighborhood safety, traffic, and crime. ${ }^{c}$ Neighborhood socioeconomic status was based on American Community Survey 5 year estimates of 2009-2013. 2013. We categorized this variable into three groups by tertiles. Higher tertiles represent higher socioeconomic status. 
As a sensitivity analysis, we conducted multiple imputation for missing data and examined the interaction effect of cultural factors in the association between the social environment and physical activity in the imputed data. Table 5 presents pooled estimates after multiple imputation. Multiple imputation changed the magnitude of coefficients but did not alter the significance.

Table 5. Results of multilevel modeling assessing associations between cultural factors and physical activity (times per week) after multiple imputation, $\mathrm{n}=3463^{\mathrm{a}}$.

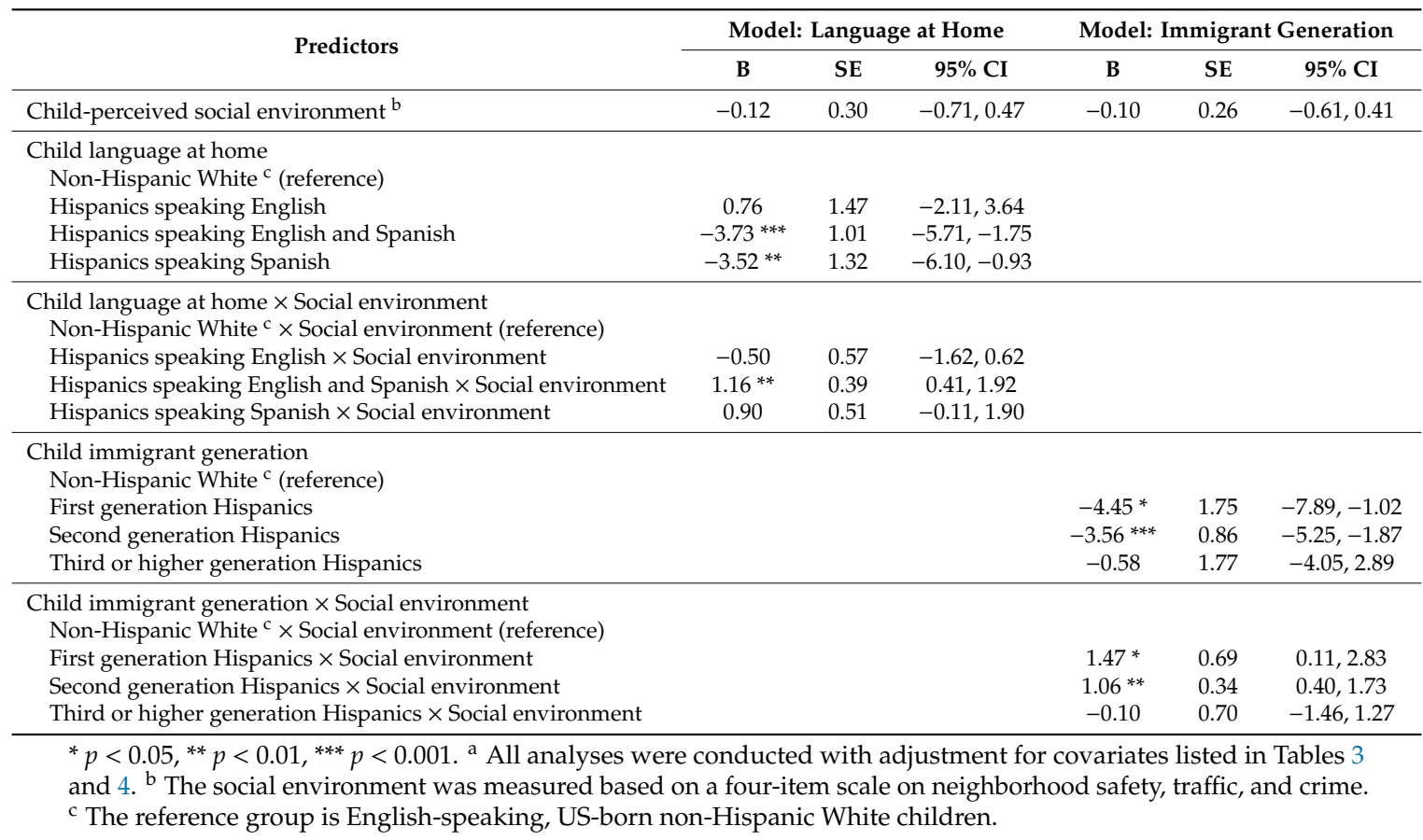

Finally, we tested the mediation of neighborhood physical activity-related social norms in the association between the social environment and physical activity. Before the mediation analysis was performed, we stratified the data by two cultural factors and tested the association between the social environment and physical activity in the stratified models. As previously reported, the association was significant in Hispanic children speaking English and Spanish, first-generation Hispanic children, and second-generation Hispanic children, but not in non-Hispanic White children, Hispanic children speaking English or Spanish, and third- or higher-generation Hispanic children. Thus, mediation analysis was performed in Hispanic children speaking English and Spanish, first-generation Hispanic children, and second-generation Hispanic children, separately. Table 6 and Figure 3 present the estimates of the effects of the social environment on physical activity. As shown in Table 6, the indirect effect estimates indicate a significant mediating role of neighborhood physical activity-related social norms for Hispanic children speaking English and Spanish $(b=0.33$ $[=0.30 \times 1.09], p<0.001)$ and second-generation Hispanic children $(b=0.40[=0.34 \times 1.17], p<0.001)$. There was no significant mediation effect of neighborhood physical activity-related social norms for first-generation Hispanic children $(b=0.09[=0.33 \times 0.29], p>0.05)$. 
Table 6. Total, direct, and indirect effects of the social environment and physical activity-related social norms on physical activity after bootstrapping.

\begin{tabular}{|c|c|c|c|c|c|c|}
\hline \multirow{2}{*}{ Pathways } & \multicolumn{2}{|c|}{ Direct Effect } & \multicolumn{2}{|c|}{ Indirect Effect } & \multicolumn{2}{|c|}{ Total Effect } \\
\hline & B & Bootstrap SE & B & Bootstrap SE & B & Bootstrap SE \\
\hline \multicolumn{7}{|l|}{ Hispanics speaking English and Spanish $(\mathrm{n}=987)$} \\
\hline Social environment $\rightarrow$ physical activity-related social norms & $0.30 * * *$ & 0.04 & - & - & $0.30 * * *$ & 0.04 \\
\hline PA-related social norms $\rightarrow$ physical activity & $1.09^{* * *}$ & 0.22 & - & - & $1.09 * * *$ & 0.22 \\
\hline Social environment $\rightarrow$ physical activity & $0.79 * *$ & 0.28 & $0.33^{* * *}$ & 0.08 & $1.12 * * *$ & 0.26 \\
\hline \multicolumn{7}{|l|}{ First generation Hispanics $(\mathrm{n}=180)$} \\
\hline Social environment $\rightarrow$ physical activity-related social norms & $0.33^{* *}$ & 0.11 & - & - & $0.33^{* *}$ & 0.11 \\
\hline PA-related social norms $\rightarrow$ physical activity & 0.29 & 0.58 & - & - & 0.29 & 0.58 \\
\hline Social environment $\rightarrow$ physical activity & $1.47 *$ & 0.70 & 0.09 & 0.19 & $1.56^{*}$ & 0.68 \\
\hline \multicolumn{7}{|l|}{ Second generation Hispanics $(n=1200)$} \\
\hline Social environment $\rightarrow$ physical activity-related social norms & $0.34^{* * *}$ & 0.04 & - & - & $0.34^{* * *}$ & 0.04 \\
\hline PA-related social norms $\rightarrow$ physical activity & $1.17^{* * *}$ & 0.21 & - & - & $1.17^{* * *}$ & 0.21 \\
\hline Social environment $\rightarrow$ physical activity & 0.35 & 0.26 & $0.40^{* * *}$ & 0.08 & $0.74^{* *}$ & 0.25 \\
\hline
\end{tabular}

${ }^{*} p<0.05,{ }^{* *} p<0.01,{ }^{* * *} p<0.001$. Note: All analyses were conducted with adjustment for covariates listed in Tables 3 and 4. 

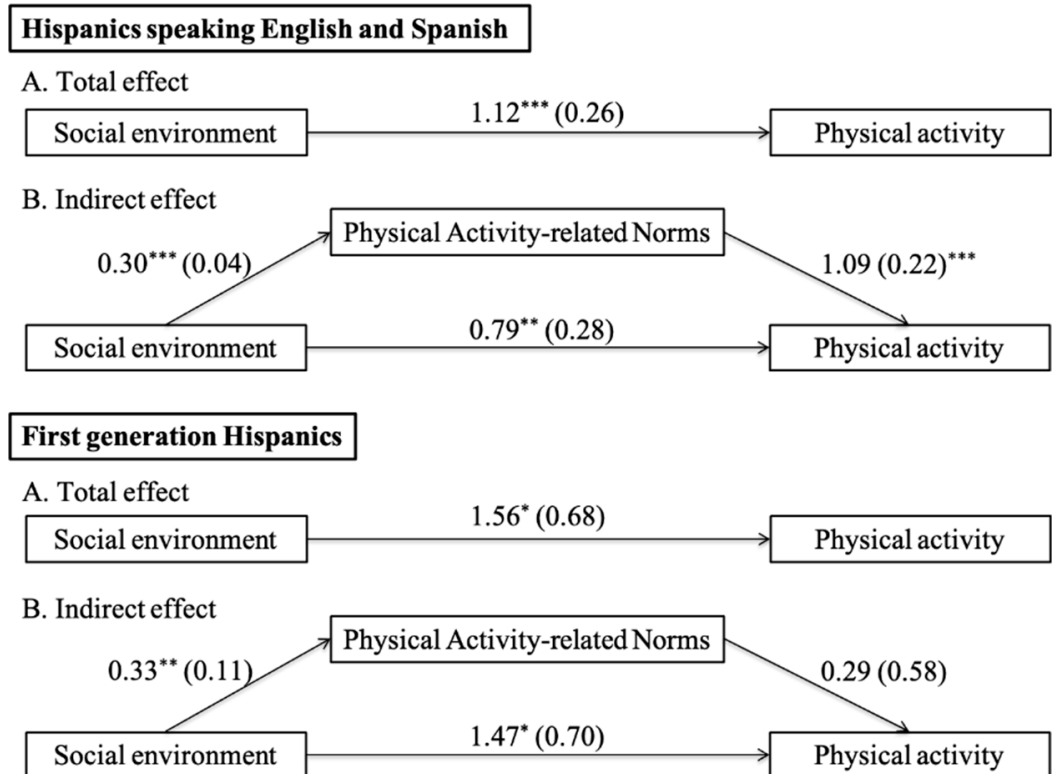

Second generation Hispanics

A. Total effect

Social environment $\quad 0.74^{* *}(0.25)$

B. Indirect effect

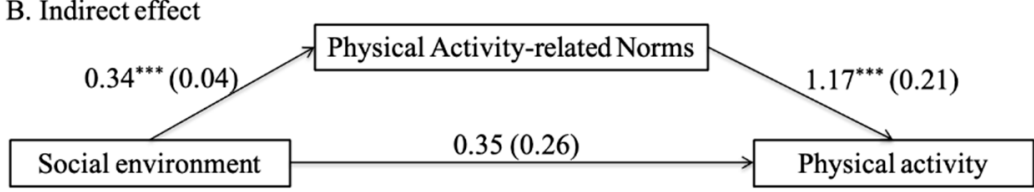

Figure 3. Path diagram for the effect of the social environment on physical activity after bootstrapping. ${ }^{*} p<0.05,{ }^{* *} p<0.01,{ }^{* * *} p<0.001$. Note: Numbers indicate unstandardized regression coefficients and standard errors in parentheses. A represents the total effect of the social environment on frequency of physical activity, and B represents the indirect effect of the social environment on frequency of physical activity through physical activity-related norms. The social environment was measured based on a four-item scale on neighborhood safety, traffic, and crime. Neighborhood physical activity-related social norms were measured based on child-reported indicators whether seeing people being active on the streets. Higher scores indicate higher quality social environment or more physical activity-promoting norms. All analyses were conducted with adjustment for covariates listed in Tables 3 and 4 .

\section{Discussion}

The present study aims to extend current knowledge by investigating mediating and moderating mechanisms by which the neighborhood social environment is associated with physical activity among non-Hispanic White children and Hispanic children. In the full sample of non-Hispanic White children and Hispanic children, the social environment was not significantly associated with physical activity. However, the association in the full sample masked subgroup differences by individual-level cultural factors. We observed that Hispanic children speaking both Spanish and English, first-generation Hispanic children, and second-generation Hispanic children had greater physical activity when living in neighborhoods with high quality social environments (vs. neighborhoods with the lowest quality social environments). For example, Hispanic children speaking Spanish and English engaged in greater physical activity by 1.11 times a week $(1.11=-0.49+1.60)$ for each unit increase in the social environment. When living in neighborhoods with the highest quality social environment, Hispanic children speaking Spanish and English showed greater physical activity than non-Hispanic White children by 1.62 times a week $(1.62=-4.78+[1.60 \times 4$ units $])$. Similar results were found in the interaction effect of immigrant generation in the association between the social environment and 
physical activity-first- and second-generation Hispanic children reported greater physical activity by 1.55 times a week $(1.55=-0.48+2.03)$ and 0.81 times a week $(0.81=-0.48+1.29)$ for each unit increase in the social environment, respectively. The results are aligned with prior research suggesting that informal support may be especially important for Hispanic children given that their families prefer to use informal support over formal support [29]. Families of first- and second-generation Hispanic children and Hispanic children speaking Spanish and English at home may experience challenges with navigating and utilizing formal support outside their neighborhood [21,30-32]. Thus, living in health-promoting neighborhoods may provide more beneficial informal support for them in comparison to non-Hispanic White children and Hispanic children from English-speaking or third-generation families who may be more likely to use formal resources. Based on these findings, we suggest that community policies focusing on change in the quality of the social environment may have a spillover influence on increasing physical activity behaviors in first- and second-generation Hispanic children and Hispanic children speaking Spanish and English.

Our results of mediation analysis showed that neighborhood physical activity-related social norms mediate the association between the social environment and physical activity in Hispanic children speaking Spanish and English and second-generation Hispanic children (Table 6 and Figure 3). It is possible that visual cues of observing neighbors and peers physically active in their neighborhoods reduce fears of exercising and leads children to accept such active, healthy lifestyles as the norm. The findings suggest that health promotion policymakers need to acknowledge the protective effect of the social environment in physical activity among Hispanic children speaking Spanish and English and second-generation Hispanic children. Also, structured neighborhood physical activity programs may help to increase physical activity participation in Hispanic children speaking Spanish and English and second-generation Hispanic children.

On the other hand, we did not observe a statistically significant mediation effect for first-generation Hispanic children. The underpinnings of our observed non-significant mediation effect for the group are not clear; one possibility is due to a small sample size issue ( $n=180$ for first-generation Hispanic children). Also, neighborhood experiences may be different across the strength of ties to Hispanic culture. First-generation Hispanic children who still hold stronger ties to Hispanic culture may have different neighborhood experiences according to the proportion of Hispanic population in the neighborhood, which was not adequately captured in this study. Future research should examine additional features of the neighborhood environment (e.g., racial/ethnic composition, social cohesion, social support, built environment) and if specific features mediate the effect of the social environment on physical activity for first-generation Hispanic children.

Important strengths of the present study include geographical diversity in the sample. In addition, Hispanic communities and families were oversampled, which led to a large number of Hispanic children in the study (1538 out of 2749 children). The main strength of our study is the investigation of the mediating and moderating mechanisms by which the neighborhood social environment is associated with physical activity, which have not been empirically tested. The potential public health significance is great given the large ethnic disparities in child physical activity in the US and the serious potential consequences of physical inactivity on child health. Our study is also subject to notable limitations. We used a cross-sectional design which prevents us from inferring causality. Due to the cross-sectional design, there is a possibility of reverse causation in which those physically active tend to choose to live in neighborhoods with high quality social environments. Longitudinal designs would elucidate the causal relation and capture the length of exposure to the neighborhood social environment and changes in the neighborhood social environment caused by residential mobility. Also, study findings are not generalizable to all US children because the HCS is not a nationally representative study despite being national in scope. Physical activity was measured using the PABR-7 instrument. The PABR-7 instrument was designed for the HCS and has been used in associational studies [36,38], but the psychometric properties have not been established [34]. Although we excluded any item explicitly asking about physical activity at school, whether physical activity occurred in a child's residential 
neighborhood was not measured. In addition, our study did not include individual-level psychological factors such as self-efficacy, motivation, and social desirability [39]. Finally, there are other factors that were not included in this analysis that may affect physical activity, such as parents' physical activity, spending time with family, psychological stress, language barriers, acculturation, enculturation, and discriminative experiences $[40,41]$. Further research should explore these various characteristics as determinants of physical activity.

\section{Conclusions}

This study showed that physical activity level varies within Hispanic children by cultural factors, and that a high-quality neighborhood social environment may serve as a resource to increase physical activity among Hispanic children speaking both Spanish and English and first- or second-generation Hispanic children. We suggest that health promotion policymakers should focus on improving the quality of neighborhood social environments as a means to encourage physical activity in children from first- and second-generation Hispanic families and those from Hispanic families speaking Spanish and English. Also, our results demonstrated that neighborhood physical activity-related social norms mediate the association between the neighborhood social environment and child physical activity in second-generation Hispanic children and Hispanic children speaking English and Spanish. The results imply that second-generation Hispanic children and Hispanic children speaking English and Spanish benefit from safe neighborhoods and from healthy social norms around physical activity. Community health programs might need to promote healthy neighborhood norms to increase physical activity in these children. Future research is warranted to include a variety of characteristics such as acculturation, discrimination, neighborhood racial/ethnic composition, and psychological factors to further understand the mechanism by which the social environment impacts physical activity in Hispanic children.

Author Contributions: Conceptualization, Y.K., N.C., L.R., and R.E.H.; methodology, Y.K., N.C., and A.L.; formal analysis, Y.K., N.C., and A.L.; writing-original draft preparation, Y.K.; writing-review and editing, Y.K., N.C., L.R., and R.E.H.; visualization, Y.K.; supervision, N.C.; funding acquisition, N.C. All authors have read and agreed to the published version of the manuscript.

Funding: Research reported in this publication was supported by the National Heart, Lung, and Blood Institute (NHLBI) of the National Institutes of Health (NIH) under award number R01HL137731 (Colabianchi, PI). The original Healthy Communities Study, was funded by the NHLBI of NIH, in collaboration with the Eunice Kennedy Shriver National Institute of Child Health and Development, National Institute of Diabetes and Digestive and Kidney Disorders, National Cancer Institute, and NIH Office of Behavioral and Social Sciences Research; Department of Health and Human Services, under award number HHSN268201000041C.

Conflicts of Interest: The authors declare no conflict of interest. The funders had no role in the design of the study; in the collection, analyses, or interpretation of data; in the writing of the manuscript, or in the decision to publish the results.

\section{References}

1. Katzmarzyk, P.T.; Denstel, K.D.; Beals, K.; Carlson, J.; Crouter, S.E.; McKenzie, T.L.; Pate, R.R.; Sisson, S.B.; Staiano, A.E.; Stanish, H. Results from the United States 2018 report card on physical activity for children and youth. J. Phys. Act. Health 2018, 15, S422-S424. [CrossRef] [PubMed]

2. Singh, G.K.; Stella, M.Y.; Siahpush, M.; Kogan, M.D. High levels of physical inactivity and sedentary behaviors among US immigrant children and adolescents. Arch. Pediatrics Adolesc. Med. 2008, 162, 756-763. [CrossRef] [PubMed]

3. Reifsnider, E.; Jeong, M.; Chatterjee, P. An ecological approach to obesity in Mexican American children. J. Pediatric Health Care 2020, 34, 212-221. [CrossRef] [PubMed]

4. Ogden, C.L.; Fryar, C.D.; Martin, C.B.; Freedman, D.S.; Carroll, M.D.; Gu, Q.; Hales, C.M. Trends in obesity prevalence by race and hispanic origin-1999-2000 to 2017-2018. Jama 2020, 324, 1208-1210. [CrossRef] 
5. United States Census Bureau Annual Estimates of the Resident Population by Sex, Age, Race Alone or in Combination, and Hispanic Origin for the United States: April 1, 2010 to July 1, 2019. Available online: https:// www.census.gov/newsroom/press-kits/2020/population-estimates-detailed.html (accessed on 22 November 2020).

6. Vespa, J.; Medina, L.; Armstrong, D.M. Demographic Turning Points for the United States: Population Projections for 2020 to 2060; U.S. Census Bureau: Washington, DC, USA, 2020.

7. Kim, Y.; Cubbin, C.; Oh, S. A systematic review of neighbourhood economic context on child obesity and obesity-related behaviours. Obes. Rev. 2019, 20, 420-431. [CrossRef]

8. Franzini, L.; Elliott, M.N.; Cuccaro, P.; Schuster, M.; Gilliland, M.J.; Grunbaum, J.A.; Franklin, F.; Tortolero, S.R. Influences of physical and social neighborhood environments on children's physical activity and obesity. Am. J. Public Health 2009, 99, 271-278. [CrossRef]

9. Nicksic, N.E.; Salahuddin, M.; Butte, N.F.; Hoelscher, D.M. Associations between parent-perceived neighborhood safety and encouragement and child outdoor physical activity among low-income children. J. Phys. Act. Health 2018, 15, 317-324. [CrossRef]

10. Goon, S.; Kontulainen, S.; Muhajarine, N. Neighborhood Built Environment Measures and Association with Physical Activity and Sedentary Time in 9-14-Year-Old Children in Saskatoon, Canada. Int. J. Environ. Res. Public Health 2020, 17, 3837. [CrossRef]

11. Musick, K.; Seltzer, J.A.; Schwartz, C.R. Neighborhood norms and substance use among teens. Soc. Sci. Res. 2008, 37, 138-155. [CrossRef]

12. Carroll-Scott, A.; Gilstad-Hayden, K.; Rosenthal, L.; Peters, S.M.; McCaslin, C.; Joyce, R.; Ickovics, J.R. Disentangling neighborhood contextual associations with child body mass index, diet, and physical activity: The role of built, socioeconomic, and social environments. Soc. Sci. Med. 2013, 95, 106-114. [CrossRef]

13. De Meester, F.; Van Dyck, D.; De Bourdeaudhuij, I.; Deforche, B.; Sallis, J.F.; Cardon, G. Active living neighborhoods: Is neighborhood walkability a key element for Belgian adolescents? BMC Public Health 2012, 12, 7. [CrossRef] [PubMed]

14. Pabayo, R.; Belsky, J.; Gauvin, L.; Curtis, S. Do area characteristics predict change in moderate-to-vigorous physical activity from ages 11 to 15 years? Soc. Sci. Med. 2011, 72, 430-438. [CrossRef] [PubMed]

15. Voorhees, C.C.; Catellier, D.J.; Ashwood, J.S.; Cohen, D.A.; Rung, A.; Lytle, L.; Conway, T.L.; Dowda, M. Neighborhood socioeconomic status and non school physical activity and body mass index in adolescent girls. J. Phys. Act. Health 2009, 6, 731-740. [CrossRef] [PubMed]

16. Perez, L.G.; Conway, T.; Arredondo, E.M.; Elder, J.; Kerr, J.; McKenzie, T.L.; Sallis, J. Where and when adolescents are physically active: Neighborhood environment and psychosocial correlates and their interactions. Prev. Med. 2017, 105, 337-344. [CrossRef]

17. Benes, D.; Dowling, J.; Crawford, S.; Hayman, L.L. Social and environmental influences on physical activity levels in Latina adolescents. Public Health Nurs. 2017, 34, 101-111. [CrossRef]

18. Crespo, N.C.; Ball, G.D.; Shaibi, G.Q.; Cruz, M.L.; Weigensberg, M.J.; Goran, M.I. Acculturation is associated with higher VO2max in overweight Hispanic children. Pediatric Exerc. Sci. 2006, 18, 89-100. [CrossRef]

19. Olvera, N.; Smith, D.W.; Lee, C.; Liu, J.; Lee, J.; Kim, J.-H.; Kellam, S.F. Comparing high and low acculturated mothers and physical activity in Hispanic children. J. Phys. Act. Health 2011, 8, S206-S213. [CrossRef]

20. Liu, J.; Probst, J.C.; Harun, N.; Bennett, K.J.; Torres, M.E. Acculturation, physical activity, and obesity among Hispanic adolescents. Ethn. Health 2009, 14, 509-525. [CrossRef]

21. Taverno, S.E.; Rollins, B.Y.; Francis, L.A. Generation, language, body mass index, and activity patterns in Hispanic children. Am. J. Prev. Med. 2010, 38, 145-153. [CrossRef]

22. Springer, A.E.; Lewis, K.; Kelder, S.H.; Fernandez, M.E.; Barroso, C.S.; Hoelscher, D.M. Physical activity participation by parental language use in 4 th, 8 th, and 11th grade students in Texas, USA. J. Immigr. Minority Health 2010, 12, 769-780. [CrossRef]

23. Williams, W.; Li, K.; Haynie, D.; Simons-Morton, B. Physical activity and sedentary behavior of US immigrant versus non-immigrant adolescents: Findings from the NEXT generation health study data. Ethn. Health 2018, 23, 329-338. [CrossRef] [PubMed]

24. Page, R.M.; Frey, J.; Talbert, R.; Falk, C. Children's feelings of loneliness and social dissatisfaction: Relationship to measures of physical fitness and activity. J. Teach. Phys. Educ. 1992, 11, 211-219. [CrossRef]

25. Ross, S.E.T.; Francis, L.A. Physical activity perceptions, context, barriers, and facilitators from a Hispanic child's perspective. Int. J. Qual. Stud. Health Well-Being 2016, 11, 31949. [CrossRef] [PubMed] 
26. Buttenheim, A.M.; Pebley, A.R.; Hsih, K.; Chung, C.Y.; Goldman, N. The shape of things to come? Obesity prevalence among foreign-born vs. US-born Mexican youth in California. Soc. Sci. Med. 2013, 78, 1-8. [CrossRef] [PubMed]

27. Finch, B.K.; Vega, W.A. Acculturation stress, social support, and self-rated health among Latinos in California. J. Immigr. Health 2003, 5, 109-117. [CrossRef] [PubMed]

28. Lara, M.; Gamboa, C.; Kahramanian, M.I.; Morales, L.S.; Hayes Bautista, D.E. Acculturation and Latino health in the United States: A review of the literature and its sociopolitical context. Annu. Rev. Public Health 2005, 26, 367-397. [CrossRef] [PubMed]

29. Ryan, S.; Ream, R.K. Variation across Hispanic immigrant generations in parent social capital, college-aligned actions, and four-year college enrollment. Am. Educ. Res. J. 2016, 53, 953-986. [CrossRef]

30. Bell, C.N.; Thorpe, R.J.; LaVeist, T.A. Race/ethnicity and hypertension: The role of social support. Am. J. Hypertens. 2010, 23, 534-540. [CrossRef] [PubMed]

31. Cardoso, J.B.; Thompson, S.J. Common themes of resilience among Latino immigrant families: A systematic review of the literature. Fam. Soc. 2010, 91, 257-265. [CrossRef]

32. Moinolmolki, N.; Gaviria-Loaiza, J.; Han, M. Immigrant families and early childhood programs: Addressing the new challenges of the 21st century. In Family Involvement in Early Education and Child Care; Emerald Group Publishing Limited: Washington, DC, USA, 2016.

33. Arteaga, S.S.; Loria, C.M.; Crawford, P.B.; Fawcett, S.B.; Fishbein, H.A.; Gregoriou, M.; John, L.V.; Kelley, M.; Pate, R.R.; Ritchie, L.D. The Healthy Communities Study: Its rationale, aims, and approach. Am. J. Prev. Med. 2015, 49, 615-623. [CrossRef]

34. Pate, R.R.P.; McIver, K.L.P.; Colabianchi, N.P.; Troiano, R.P.P.; Reis, J.P.P.; Carroll, D.D.P.; Fulton, J.E.P. Physical activity measures in the Healthy Communities Study. Am. J. Prev. Med. 2015, 49, 653-659. [CrossRef] [PubMed]

35. Diez-Roux, A.V.; Merkin, S.S.; Arnett, D.; Chambless, L.; Massing, M.; Nieto, F.J.; Sorlie, P.; Szklo, M.; Tyroler, H.A.; Watson, R.L. Neighborhood of Residence and Incidence of Coronary Heart Disease. N. Engl. J. Med. 2001, 345, 99-106. [CrossRef] [PubMed]

36. Kim, Y.; Landgraf, A.; Colabianchi, N. Living in High-SES Neighborhoods Is Protective against Obesity among Higher-Income Children but Not Low-Income Children: Results from the Healthy Communities Study. J. Urban Health 2020, 97, 175-190. [CrossRef] [PubMed]

37. Gunzler, D.; Chen, T.; Wu, P.; Zhang, H. Introduction to mediation analysis with structural equation modeling. Shanghai Arch. Psychiatry 2013, 25, 390.

38. Kaczynski, A.T.; Besenyi, G.M.; Child, S.; Morgan Hughey, S.; Colabianchi, N.; McIver, K.L.; Dowda, M.; Pate, R.R.; Team, H.C.S. Relationship of objective street quality attributes with youth physical activity: Findings from the Healthy Communities Study. Pediatric Obes. 2018, 13, 7-13. [CrossRef]

39. Craggs, C.; Corder, K.; van Sluijs, E.M.; Griffin, S.J. Determinants of change in physical activity in children and adolescents: A systematic review. Am. J. Prev. Med. 2011, 40, 645-658. [CrossRef]

40. Korcz, A.; Krzysztoszek, J.; Łopatka, M.; Ludwiczak, M.; Górska, P.; Bronikowski, M. The role of family time together in meeting the recommendation for physical activity among primary school children. Int. J. Environ. Res. Public Health 2020, 17, 3970. [CrossRef]

41. Sigmundová, D.; Sigmund, E.; Badura, P.; Hollein, T. Parent-Child Physical Activity Association in Families With 4-to 16-Year-Old Children. Int. J. Environ. Res. Public Health 2020, 17, 4015. [CrossRef]

Publisher's Note: MDPI stays neutral with regard to jurisdictional claims in published maps and institutional affiliations.

(C) 2020 by the authors. Licensee MDPI, Basel, Switzerland. This article is an open access article distributed under the terms and conditions of the Creative Commons Attribution (CC BY) license (http://creativecommons.org/licenses/by/4.0/). 